\title{
Percolation thresholds in chemical disordered excitable media
}

\author{
I. Sendiña-Nadal, ${ }^{1, *}$ D. Roncaglia, ${ }^{2}$ D. Vives,${ }^{3}$ V. Pérez-Munuzuri, ${ }^{1}$ M. Gómez-Gesteira, ${ }^{1}$ V. Pérez-Villar, ${ }^{1}$ J. Echave, ${ }^{2}$ \\ J. Casademunt, ${ }^{4}$ L. Ramírez-Piscina, ${ }^{5}$ and F. Sagués ${ }^{3}$ \\ ${ }^{1}$ Group of Nonlinear Physics, Faculty of Physics, University of Santiago de Compostela, 15706 Santiago de Compostela, Spain \\ ${ }^{2}$ Centro de Estudios e Investigaciones (CEI), Universidad Nacional de Quílmes, Saenz Peña 180, 1876 Bernal, Argentina \\ ${ }^{3}$ Departament de Química Física, Universitat de Barcelona, Diagonal 647, 08028 Barcelona, Spain \\ ${ }^{4}$ Departament d'Estructura i Constituents de la Matèria, Universitat de Barcelona, Diagonal 647, 08028 Barcelona, Spain \\ ${ }^{5}$ Departament de Física Aplicada, Universitat Politècnica de Catalunya, Gregorio Marañón 44, 08028 Barcelona, Spain
}

(Received 14 April 1998)

\begin{abstract}
The behavior of chemical waves advancing through a disordered excitable medium is investigated in terms of percolation theory and autowave properties in the framework of the light-sensitive Belousov-Zhabotinsky reaction. By controlling the number of sites with a given illumination, different percolation thresholds for propagation are observed, which depend on the relative wave transmittances of the two-state medium considered. [S1063-651X(98)51008-2]
\end{abstract}

PACS number(s): 05.70.Ln, 03.40.Kf

Percolation theory allows us to interpret many physical experiments where connectivity plays a fundamental role (for a review, see Ref. [1]). Representative examples are electrical conductivity in disordered semiconductors [2,3], percolation at the superconducting transition in $\mathrm{Y}-\mathrm{Ba}-\mathrm{Cu}-\mathrm{O}$ thin films [4], two-dimensional percolation in cluster deposition [5], and fingering phenomena [6].

Considered in its original version, percolation is a static geometric tool relating accessibility properties within a lattice and the eventual crossing of it to the occupancy probability of its constituent sites. In this sense, percolation theory establishes the existence of a threshold or critical value $p_{c}$, below which only finite (nonconnecting) clusters are present, whereas an infinitely connected path is generated at $p_{c}$. In addition to the examples cited above, there are well-known situations, such as fluid displacement in oil fields or forest fire spreading, where percolation ideas, even at their most simple and intuitive level, have been fruitfully used [1]. However, as far as we know percolation concepts have never been systematically addressed before in connection with autowave propagation in chemical media. This is quite surprising if one considers the apparent similarities between some of these situations and the invasion of a nonexcited medium by an excited front.

On the other hand, the role of excitation inhomogeneities has been an issue of great interest in the last few years both theoretically and experimentally. In this respect, wave front propagation in nonhomogeneous media has been studied from different points of view: the interaction of waves and inert obstacles (which is especially relevant in cardiology, since reentries can be anchored [7-9] or planar fronts broken $[10-13])$ or by the role of modulations $[14,15]$ and fluctuations on patterns of distributed excitability [16-19].

In this paper, we study both experimentally and numerically the propagation of a wave front on a medium consisting

\footnotetext{
*Author to whom correspondence should be addressed. Electronic address: irene@fmmeteo.usc.es
}

of clusters of two excitabilities. Waves can only propagate through one kind of cluster, which reminds us of the situation found in experiments of disordered semiconductors $[2,3]$. Characteristic times are studied as a function of the relative excitability levels, and interpretations are given in terms of the classical percolation theory.

Experiments were performed with the BelousovZhabotinsky reagent catalyzed by the ruthenium bipyridyl complex $\mathrm{Ru}$ (bpy), which is sensitive to visible light, thus allowing experimental control of the excitability of the system [20-24]. The Ru complex was immobilized in a silicagel matrix (using a solution of $15 \%$ sodium silicate, $0.71 \mathrm{~m} M \mathrm{Ru}(\mathrm{bpy})_{3}^{2+}$ and $0.18 M \mathrm{H}_{2} \mathrm{SO}_{4}$; preparation is the same as in [25]) in a Petri dish (diameter, $14 \mathrm{~cm}$; thickness, $1 \mathrm{~mm}$ ). The solution (initial concentrations: $0.18 \mathrm{M}$ $\mathrm{KBr}, 0.33 M$ malonic acid, $0.39 M \quad \mathrm{NaBrO}_{3}$, and $0.69 \mathrm{M} \mathrm{H}_{2} \mathrm{SO}_{4}$ ) was poured onto the gel. The temperature was kept constant at $25 \pm 1{ }^{\circ} \mathrm{C}$. White light $190 \mathrm{~W}$ halogen lamp) first passed a diffusion screen, then the Petri dish, the interference filter ( $450.6 \mathrm{~nm}$; transmission 56\%), and, finally, video equipment for image recording [26,27].

In order to introduce a spatial variation on the wave propagation conditions of the inhomogeneous medium, a transmission function $T(x, y)$ was printed out on a transparency, which was placed between the light source and the Petri dish [14,19]. The prescribed distribution $T(x, y)$ directly fixes the light intensity reaching the medium (medium excitability decreases as light intensity increases $[14,28])$. In the situation considered here, $T(x, y)$ reproduces a dichotomous random distribution of squares. The size of the squares was larger than the characteristic width of the front.

The typical experimental procedure consisted in generating a planar wave front at the bottom side of the medium and monitoring its evolution until it either reached the top side or it disappeared. Figure 1 shows the time evolution of a single experiment for a given proportion of dark squares. In Fig. 1(a) the planar front was initiated at the bottom of the medium. The front breaks up into several parts that propagate 


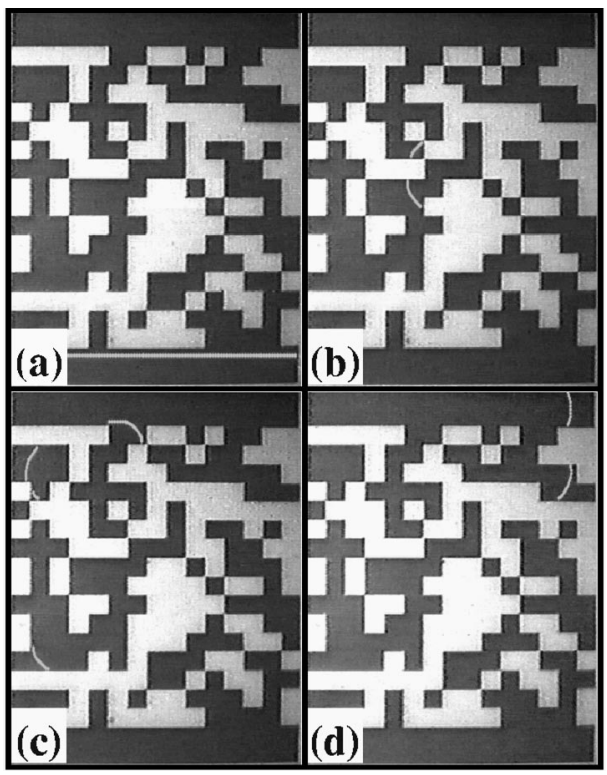

FIG. 1. Four different snapshots of the propagation of a wave front through a light sensitive medium consisting of a random distribution of excitable squares (black squares) in a nonexcitable medium (white squares). The wave can propagate only through black sites. The initial wave front propagating from the bottom, (a), breaks up and only a small part reaches the top while other fronts move around the lattice, (b)-(d). Times: (a) $0 \mathrm{~s}$, (b) $778 \mathrm{~s}$, (c) 1068 $\mathrm{s}$, and (d) $1405 \mathrm{~s}$. [Proportion of black squares, $p=0.5$; size of squares, $3 \mathrm{~mm}$; medium size, $4.5 \mathrm{~cm} \times 6 \mathrm{~cm}$; light intensity through black squares $\left(250 \mu \mathrm{W} / \mathrm{cm}^{2}\right)$, and through white squares $\left(750 \mu \mathrm{W} / \mathrm{cm}^{2}\right)$.]

along the lattice, as shown in Figs. 1(b)-1(c). Only one of them reaches the upper side first, whereas the others wander around the lattice until their total annhilation [Fig. 1(d)].

The experimental measurements of transit and termination times were done for different values of the proportion $p$ of squares with a given transmittance (excitability). Owing to the fact that the medium is finite, and in order to compare the results with theoretical predictions, five experiments were carried out for each value of $p$. Each realization corresponds to a different inhomogenous pattern of $n \times n$ randomly distributed dark and light squares (black and white from now on) of transmittances $T_{b}$ and $T_{w}$, respectively, chosen in such a way that the wave cannot propagate through the white squares. This favors the breaking up of the front into several small fronts that move independently through the lattice. In order to change the actual connectivity of the twodimensional lattice, two light intensities from the light source were used. With the lowest light intensity, waves can propagate both from a black site to the nearest neighbor black squares (squares with one side in common) and to the nextnearest black neighbors (squares with one vertex in common). With the highest light intensity, waves can only transit between two black sites with a common side.

Numerical simulations were performed with a twovariable Oregonator model modified to include light-induced bromide production [29], which in dimensionless form reads

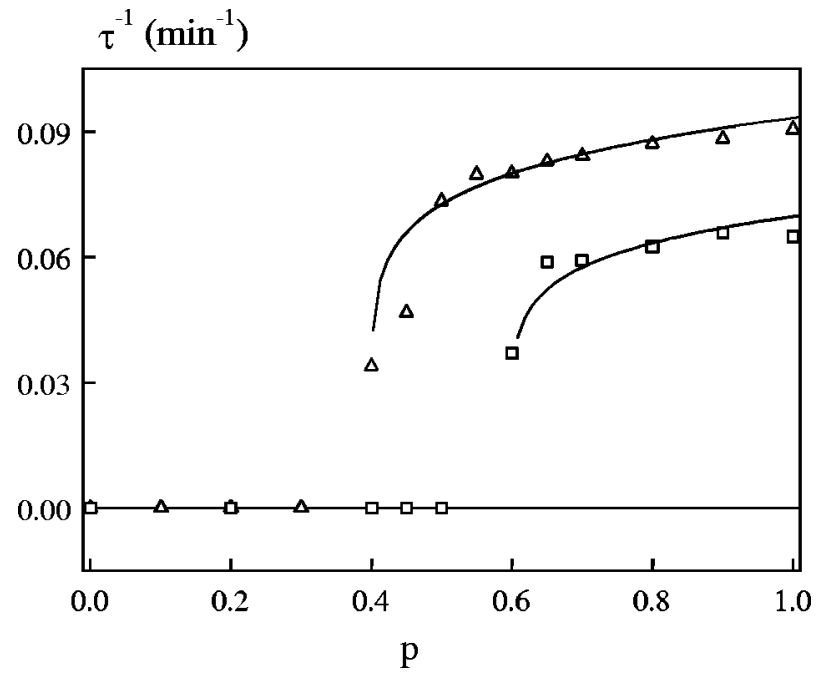

FIG. 2. Inverse of the transit time for propagation between both edges of the experimental system (see text) is plotted as a function of the probability of black sites $p$. The continuous lines represent nonlinear fits to Eq. (2) above the percolation threshold for two different geometries: $\square$, cells connected by common sides; $\triangle$, cells connected by common sides and corners. At least five experiments were carried out for each value of $p$ in order to compare with theoretical results typical of infinite media. Parameters of the fitting curves are $\triangle, \alpha=0.1, p_{c}=0.4 ; \square, \alpha=0.08, p_{c}=0.6$.

$$
\begin{gathered}
\frac{\partial u}{\partial t}=\frac{1}{\varepsilon}\left(u-u^{2}-[f v+\phi(x, y)] \frac{u-q}{u+q}\right)+D_{u} \nabla^{2} u, \\
\frac{\partial v}{\partial t}=(u-v)+D_{v} \nabla^{2} v,
\end{gathered}
$$

where $u$ and $v$ describe $\mathrm{HBrO}_{2}$ and catalyst concentrations, respectively. $D_{u}$ and $D_{v}$ are the diffusion coefficients of both variables. $f, q$, and $\varepsilon$ are parameters related to the kinetics of the Belousov-Zhabotinsky reaction. $\phi(x, y)$ represents the light-induced flow of $\mathrm{Br}^{-}$and it is assumed to be directly proportional to $T(x, y)$. The system was composed of 40 $\times 40$ square cells, each one of (dimensionless) length ten, and periodic boundary conditions in the lateral direction were imposed. We employed an explicit finite-difference scheme with mesh size 0.3 and time step 0.001 . To reduce finite-size statistical dispersion, the results were typically averaged over 10 runs for each prescribed level of excitability in the medium.

The motion of the wave front has the same features as in the typical models in the percolation of forest fires or disordered semiconductors $[1,3]$. No successful wave propagation is found below a critical value $p_{c}$, which depends on the light intensity used ( $p_{c} \approx 0.4$ or $p_{c} \approx 0.6$ ), as is shown in Fig. 2. For $p$ well below $p_{c}$, there are mostly isolated sites and a few pairs and other small clusters, so that the wave cannot reach the upper edge of the pattern. For $p$ well above $p_{c}$, most of the black sites belong to the infinite cluster, and the wave velocity is approximately constant. At the percolation threshold $p_{c}$ a fractal path of neighboring black squares appears that connects the bottom with the top of the medium, 

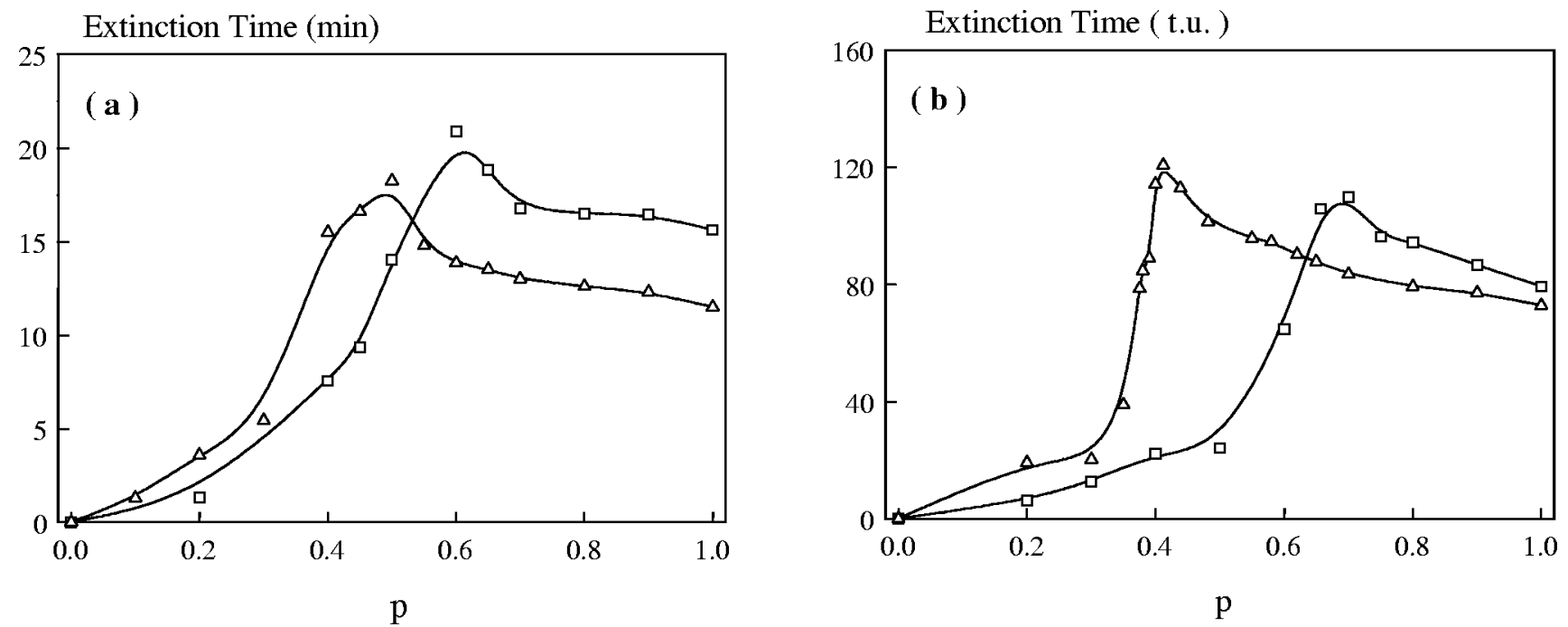

FIG. 3. Extinction time for autowaves propagating through lattices with probability of black sites $p$ : (a) experimentally and (b) numerically, for the two different lattice geometries (symbols have the same meaning as in Fig. 2). For $p \rightarrow 0$, the front can propagate only through a few black sites before extinction, while for $p \rightarrow 1$, almost the whole front reaches the top of the lattice. In between, at the cusp the small broken parts of the front spend an infinite time (not in the experiment, but in an infinite lattice) wandering through the lattice before annhilation. Solid lines are spline interpolations of the experimental and numerical data. (a) Experiments: maxima located at $\triangle, p_{c} \approx 0.5$; $\square$, $p_{c}=0.6$. Parameters are as in Fig. 1. (b) Simulations: maxima located at $\triangle, p_{c} \approx 0.45 ; \square, p_{c}=0.6$. Model parameters: $f=3, q=0.002$, $\varepsilon=0.05, D_{u}=1, D_{v}=0, \phi_{b}=0.01(0.015)$, and $\phi_{w}=0.04(0.04)$ to reproduce $p_{c} \approx 0.45\left(p_{c}=0.6\right)$. t.u. $=$ time unit.

either through the corners or common sides $\left(p_{c} \approx 0.4\right)$, or only through the common sides $\left(p_{c} \approx 0.6\right)$. As a result, even though the wave propagates through the medium, the percolating cluster is very different from a straight line, slowing down the wave front velocity. Experimental results corresponding to the elapsed time to transit between both edges for $p>p_{c}$ were fitted to a power law, which is typical of critical phenomena, and for a two-dimensional lattice can be easily calculated [1] as

$$
\tau^{-1}=\alpha\left(p-p_{c}\right)^{\beta}
$$

with the critical exponent $\beta=5 / 36$. (Zero values of the inverse of such an elapsed time naturally correspond to unsuccessful propagation.)

Although the lattice was square shaped, light intensity allowed us to select the way that the wave front moved through clusters, either through corners and sides or through sides only. This changes the connectivity of effective neighbors on a given black site from eight to four. Thus, the percolation threshold should vary from 0.5928 to 0.4072 [1] as reproduced by the results of the figure.

The extinction time of the autowave is defined as the time elapsed until termination is reached, which is averaged over many lattices with the same $p$. Figures 3(a) and 3(b) show the lifetime of autowaves as a function of the probability $p$ for the two spatial geometries: square lattice connected only by nearest neighbors, and by both nearest and next-nearest ones, both experimentally and numerically. Experiments show a peak at $p \approx 0.6$ for the first geometry and near $p$ $\approx 0.5$ for the second one. At $p \approx 0.6$ extinction times are longer than at $p \approx 0.5$ because in the experiments light inten- sity was kept higher in order to restrict wave propagation through sides that were in common only, thus decreasing wave velocity. The presence of such peaks is associated with the fractal properties of the connecting cluster present at $p$ $=p_{c}$, whose length (and hence the corresponding transit time) is no longer linearly proportional to the length of the lattice. Due to finite-size effects the peaks appear as being of finite height, and with a rounded shape. In fact, the value of $p \approx 0.5$ does not match exactly the threshold $p_{c}=0.4072$ predicted by the theory and found in the transit times measurements of Fig. 2. However, in the simulations lattice sizes were larger, and the critical value obtained in Fig. 3(b) $\left(p_{c}\right.$ $\approx 0.4$ ) approached much better to the expected value than in the experimental case.

In this paper, we have presented results of autowave propagation on a lattice of excitable and nonexcitable clusters. The proportion $p$ of black sites was varied from zero to one in order to study percolation effects on wave propagation. The effective wave front velocity (proportional to the inverse of the transit time) is observed to jump from zero to finite values at a threshold $p=p_{c}$ (very close to the percolation thresholds expected for a square lattice) when, as predicted by the classical percolation theory, a cluster of sites with the same excitability spans the medium. These thresholds depend on the number of effective neighbors.

This work was partially supported by the Comisión Interministerial de Ciencia y Tecnología under Project Nos. DGES-PB96-0937, PB96-1001, and PB96-0241, and Comissionat per a Universitats i Recerca (Generalitat de Catalunya). Calculations were performed at the Supercomputation Center of Galicia (CESGA) and Catalunya (CESCA), Spain. D. Vives benefited from an FI grant from Generalitat de Catalunya. 
[1] D. Stauffer and A. Aharony, Introduction to Percolation Theory (Taylor \& Francis, London, 1994); M. Sahimi, Applications of Percolation Theory (Taylor \& Francis, London, 1994).

[2] T.P. Eggarter and M.H. Cohen, Phys. Rev. Lett. 25, 807 (1970).

[3] B.J. Last and D.J. Thouless, Phys. Rev. Lett. 27, 1719 (1971).

[4] L.B. Kiss, P. Svedlindh, L. Lundgren, J. Hudner, H. Ohlsen, and L. Stolt, Solid State Commun. 75, 747 (1990).

[5] P. Jensen et al., Physica A 185, 104 (1992).

[6] U. Oxaal et al., Nature (London) 329, 32 (1987).

[7] G.R. Mines, Trans. R. Soc. Can. 4, 43 (1914).

[8] M.A. Allesie, F.M.I. Bonke, and T.Y.G. Scopman, Circ. Res. 33, 54 (1973).

[9] M.S. Spach, W.T. Miller, D.B. Geselowitz, R.C. Borr, J.M. Kootsey, and E.A. Johnson, Circ. Res. 48, 39 (1981).

[10] A.T. Winfree, J. Theor. Biol. 40, 45 (1973).

[11] A. Karma, Phys. Rev. Lett. 71, 1103 (1993).

[12] M. Gómez-Gesteira, J.L. del Castillo, M.E. Vázquez-Iglesias, V. Pérez-Muñuzuri, and V. Pérez-Villar, Phys. Rev. E 50, 6 (1994).

[13] J.M. Starobin and C.F. Starmer, Phys. Rev. E 54, 430 (1996).

[14] I. Sendiña-Nadal, M. Gómez-Gesteira, V. Pérez-Munuzuri, V. Pérez-Villar, J. Armero, L. Ramírez-Piscina, J. Casademunt, F. Sagués, and J.M. Sancho, Phys. Rev. E 56, 6298 (1997).

[15] J. Armero, A.M. Lacasta, L. Ramírez-Piscina, J. Casademunt, J.M. Sancho, and F. Sagués, Europhys. Lett. 33, 429 (1996); Phys. Rev. E 56, 5405 (1997).
[16] P. Jung and G. Mayer-Kress, Phys. Rev. Lett. 74, 2130 (1996); Chaos 5, 458 (1995).

[17] J. Maselko and K. Showalter, Physica D 49, 21 (1991).

[18] S. Kádar, J. Wang, and K. Showalter, Nature (London) 391, 770 (1998)

[19] I. Sendiña-Nadal, A.P. Pérez-Muñuzuri, D. Vives, V. PérezMunuzuri, J. Casademunt, L. Ramírez-Piscina, J.M. Sancho, and F. Sagués, Phys. Rev. Lett. 80, 5437 (1998).

[20] J.N. Demas and D. Diemente, J. Chem. Educ. 50, 357 (1973).

[21] V. Gaspar, G. Bazsa, and M.T. Beck, Z. Phys. Chem. 264, 43 (1983).

[22] L. Kuhnert, Nature (London) 319, 393 (1986).

[23] L. Kuhnert, K.I. Agladze, and V.I. Krinsky, Nature (London) 337, 224 (1989).

[24] M. Markus, Zs. Nagy-Ungvaray, and B. Hess, Science 257, 225 (1992)

[25] T. Yamaguchi, L. Kuhnert, Zs. Nagy-Ungvaray, S.C. Müller, and B. Hess, J. Phys. Chem. 95, 5831 (1991).

[26] A.P. Muñuzuri, V.A. Davydov, M. Gómez-Gesteira, V. PérezMuñuzuri, and V. Pérez-Villar, Phys. Rev. E 54, R5921 (1996).

[27] A.P. Munuzuri and M. Markus, Phys. Rev. E 55, 33 (1997).

[28] M.K. Ram Reddy, Zs. Nagy-Ungvarai, and S.C. Müller, J. Phys. Chem. 98, 12255 (1994).

[29] H.J. Krug, L. Pohlmann, and L. Kuhnert, J. Phys. Chem. 94, 4862 (1990). 